\title{
Adaptive Opportunistic Cooperative Control Mechanism Based on Combination Forecasting and Multilevel Sensing Technology of Sensors for Mobile Internet of Things
}

\author{
Yong Jin, ${ }^{1}$ Kaijian Xia, ${ }^{2}$ Xiaogang Ren, ${ }^{2}$ Jian Cai, ${ }^{2}$ and Hongchun Fan ${ }^{2}$ \\ ${ }^{1}$ School of Computer Science \& Engineering, Changshu Institute of Technology, Changshu 215500, China \\ ${ }^{2}$ Changshu No. 1 People's Hospital, Changshu, Jiangsu 215500, China \\ Correspondence should be addressed to Kaijian Xia; xiakaijian@163.com
}

Received 15 June 2014; Revised 26 July 2014; Accepted 1 August 2014; Published 17 August 2014

Academic Editor: Junjie Chen

Copyright (C) 2014 Yong Jin et al. This is an open access article distributed under the Creative Commons Attribution License, which permits unrestricted use, distribution, and reproduction in any medium, provided the original work is properly cited.

\begin{abstract}
In mobile Internet of Things, there are many challenges, including sensing technology of sensors, how and when to join cooperative transmission, and how to select the cooperative sensors. To address these problems, we studied the combination forecasting based on the multilevel sensing technology of sensors, building upon which we proposed the adaptive opportunistic cooperative control mechanism based on the threshold values such as activity probability, distance, transmitting power, and number of relay sensors, in consideration of signal to noise ratio and outage probability. More importantly, the relay sensors would do self-test real time in order to judge whether to join the cooperative transmission, for maintaining the optimal cooperative transmission state with high performance. The mathematical analyses results show that the proposed adaptive opportunistic cooperative control approach could perform better in terms of throughput ratio, packet error rate and delay, and energy efficiency, compared with the direct transmission and opportunistic cooperative approaches.
\end{abstract}

\section{Introduction}

In mobile Internet of Things, the sensing, collection, processing of wireless data, and so forth are relying on the highdensity deployment sensors. However, due to the constraints and challenges of sensors $[1,2]$, for example, dynamic wireless link, limited bandwidth, susceptible to interference channel, limited energy and computation capability, and limited storage, it is difficult to guarantee the quality of service (QoS) for mobile Internet of Things application (MIOT).

The random variation $[3,4]$ of sensors and Internet of Things (IOT) seriously especially affect the system performance such as mobility, activity probability, and channel quality. There is one key issue in data transmission over MIOT, when and how to build the optimal cooperative data communication on the basis of the sensors and network state.

In this paper, we investigate how and when to choose the optimal sensors with some threshold values based on forecasting, to build the reliable, real time, high resources efficiency and effective data transmission control progress, and to satisfy the diversity requirements of MIOT services.
The rest of the paper is organized as follows. The related work and our work object are elaborated in Section 2. Section 3 describes the randomness verification of data control events sequences. In Section 4, we design a combination forecasting approach based on multilevel sensing technology of sensors in MIOT and give the analysis foundation to obtain the threshold. The proposed scheme of opportunistic cooperative control approach is used to guarantee the real time, high efficient, and reliable data transmission in MIOT, which is shown in Section 5. Section 5 also proposes the adaptive opportunistic cooperative control mechanism based on combination forecasting, including the details of implementation. Mathematics results are given in Section 6. Finally, we conclude the paper in Section 7.

\section{Related Work}

Internet of Things relies on collaboration between the highdensity data-sensing deployment sensors, to implement the collection and processing $[5,6]$; however sensors in IOT 
are self-limiting size, energy, data storage, processing power, and wireless bandwidth, so the cooperative data transmission progress has to consider these factors.

On the one hand, it is well known that sensors could effectively improve the utilization of system resources and network performance. However, there are some key problems of cooperative communication over MIOT [7, 8], including when to start cooperative communication, which sensors to join the cooperative process, and how to establish cooperative transmission control mechanisms. Christophe proposed the approach of such vision based on a federated network comprised of sensors [9]. In view of cluster and multichannel media access control (MAC) schemes, Jonghun Kim and Lee overcome interference with group mobility for reliable wireless communication services over IOT [10].

On the other hand, the status of sensors, which would be affected by transmitting power, channel quality, distance, or activity probability, could influence directly the performance of cooperative communication. So the cooperative transmission process should be rebuilt adaptively and promptly. In [11], authors divided the wireless channels according to implicit ACK and proposed the protocol, which is able to select the routing path in accordance with the link quality. The simple and robust ZigBee transceiver [12] was designed, which is able to support transmission distance of $1.2 \mathrm{~km}$ and moving speed of $60 \mathrm{~km} / \mathrm{h}$. In the study of article [13], the energy consumption of multi-path-optimized link state routing and dynamic MANET on demand were compared and analyzed.

Particularly, in the progress of opportunistic cooperation, how to effectively reduce energy consumption in the Internet of Things for sensors is one key problem, which is used to improve system throughput, transmission delay, and reliability. Since our previous research introduced the Markov chain model based on Automatic Repeat reQuest (ARQ) and hybrid ARQ to study the characteristics of quality of service and designed the quality of service supported strategy with dynamic priority [14], energy-efficiency-aware adaptive relay selection algorithm based on signal to noise ratio (SNR) was presented to achieve higher system throughput and lower delay [15]. The author of [16] characterized the bidirectional effects between human and opportunistic IOT and discussed the technical challenges and then proposed the reference architecture for developing opportunistic IOT systems. Rosárioa et al. proposed the link quality and geographical beaconless OR protocol for efficient video dissemination for mobile multimedia Internet of Things [17]. Gregory Katsaros et al. defined one new collaboration domain and presented the framework through which opportunistic collaboration services can be provisioned.

In a word, because of the special physical and electrical properties of sensors and dynamic Internet of Things topology, especially the diversity of application service in complex environments, it is difficult to meet the needs of practical application and diversity to guarantee quality of service by using direct transmission or fixed cooperative transmission control method. Therefore, it is necessary and important to consider and research the adaptive opportunistic multilevel sensing prediction method, which can not only predict the
TABLE 1: Results of randomness verification.

\begin{tabular}{ccccccccc}
\hline$m$ & & & \multicolumn{2}{c}{$\partial$} \\
& 0.01 & 0.02 & 0.05 & 0.1 & 0.2 & 0.3 & 0.4 & 0.5 \\
\hline 4 & T & T & T & F & F & F & F & F \\
5 & T & T & T & $T$ & F & F & F & \\
6 & T & T & T & $T$ & $\mathbf{T}$ & F & F & F \\
7 & T & T & T & $T$ & T & F & F & F \\
8 & T & T & T & $T$ & T & F & F & F \\
\hline
\end{tabular}

Here, $T$ denotes the characteristic of event sequence randomness. $F$ denotes that the event sequence does not have the random nature. Bold zone represents the sequence which has the random nature when $m$ is from 4 to 8 and $\partial$ is from 0.01 to 0.05 . Italic zone represents the sequence which has the random nature when $m$ is from 5 to 8 and $\partial$ is 0.1 . Bold italic zone represents the sequence which has the random nature when $m$ is from 6 to 8 and $\partial$ is 0.2 .

future trend of Internet of Things, but also establish the best opportunistic cooperation transmission.

\section{Randomness Verification of Events Sequences}

In IOT, data is transmitted in a certain time interval, the event control process in accordance with the arrangement order of events sequence. The dynamic features of the collected event sequence could be analyzed by the correlation coefficients $m$ with significance level $\partial$, which is used to judge whether the sequence satisfies the random characteristic for data transmission control event prediction.

Let $X$ denote the collected data sequence; the correlation coefficients could be obtained by

$$
m=\frac{\sum_{t=1}^{N-k}\left(x_{t}-\bar{x}\right)\left(x_{t+k}-\bar{x}\right)}{\sum_{t=1}^{N}\left(x_{t}-\bar{x}\right)^{2}} .
$$

Here, $\bar{x}=(1 / N) \sum_{t=1}^{N} x_{t}$.

If $m$ satisfies the following inequality, the events sequence should have characteristics of randomness:

$$
|m| \leq \frac{T_{a}}{\sqrt{N}}
$$

Here, $T_{a}$ denotes the weight coefficient according to the significance level $\partial$.

In order to analyze the dynamic characteristics of $m$ and $\partial$ with the events sequences, $m$ is set from 4 to 8 ; the step is 1 . $\partial$ is set to one of the matrices [0.01, 0.02, 0.05, 0.1, 0.2, $0.3,0.4,0.5]$. The results are shown in Table 1 .

The following conclusions could be obtained from Table 1.

(1) The event sequence has the random nature when $\partial$ is less than or equal to 0.05 .

(2) The event sequence has the random nature when $m$ is greater than or equal to 5 and $\partial$ is 0.1 .

(3) The event sequence has the random nature when $m$ is greater than or equal to 6 and $\partial$ is 0.2 .

(4) The event sequence does not have the random nature when $\partial$ is greater than or equal to 0.3 . 


\section{Multilevel Combination Forecasting Approach Based on Sensing}

When data communications of IOT were affected by cyclical movements, dynamic topology, and random interference, it is difficult to predict wireless transmission events. So, the weighted moving average method could be used to eliminate these confounding factors, in order to clear the transmission control event trend for effective forecasting.

The $n$ statistical packet loss time from the IOT as the base for the actual communication process was used to predict the next packet loss time. Event prediction value could be calculated by the weighted moving average prediction value events according to

$$
\mathrm{WMA}_{t+1}=\frac{1}{n} \sum_{i=t+1-n}^{t} w_{i-t+n} x_{i} .
$$

Here, $\mathrm{WMA}_{t+1}$ denotes the weighted moving average after $t$ cyclical movements. $w$ denotes weighted weights.

Then, the $n+1$ and $n+2$ packet loss time would be predicted according to $n$ statistical values, which is used to evaluate the reliability and real time performance of this approach. Here, $n$ is set from 6 to $10 . w$ is set to the following three sets of data: (1) $w_{1}=[0.05,0.1,0.5,1.0,1.5]$, (2) $w_{2}=$ $[0.5,1.0,1.5,2.0,2.5]$, and $(3) w_{3}=[0.05,0.1,0.5,1.0]$. The prediction results are shown as in Figure 1 and Table 2.

The real time performance analysis of weighted moving average method was summarized in Table 2 and Figure 1 as follows.

(1) The blue area represents the predicted time for different parameter settings.

(2) Light green represents the 11th actual statistics; wine red represents the 12 th actual statistics.

(3) The real time performance was better when $n$ is smaller. That means that the prediction value is closer to the actual predictive value.

Meanwhile, the reliability analysis is shown in Figure 2, and the conclusions are as follows.

(1) When $n$ is small the weighted weights must be not too small; otherwise error would be too large.

(2) When $n$ is greater and the error is fewer, the reliability is higher.

(3) The appropriate weighted weights should be selected based on the forecast demand.

(4) Increasing the length of the array could reduce the prediction error and improve reliability when each element of weighted weight array is equal.

Diversity sensing weighted moving average method is able to choose the best predictive parameter setting to analyze $n$ and $w$ for prediction with high reliability and real time performance, but it must have $n$, the past actual statistics. It is difficult to meet this condition especially at the early time of Internet of Things, which has to store large amounts of data and would increase the burden on the system.
TABLE 2: Comparative analysis weighted moving average forecast.

\begin{tabular}{lccc}
\hline Parameter settings & Actual value & Predictive value & Error \\
\hline$\left(n_{1}=6, w_{1}\right)$ & 39 & 43.5282 & 0.1492 \\
$\left(n_{1}=6, w_{2}\right)$ & 39 & 46.7233 & 0.2866 \\
$\left(n_{1}=6, w_{3}\right)$ & 39 & 45.1210 & 0.1676 \\
$\left(n_{2}=10, w_{1}\right)$ & 48.5 & 50.7251 & 0.1014 \\
$\left(n_{2}=10, w_{2}\right)$ & 48.5 & 52.1369 & 0.1573 \\
$\left(n_{2}=10, w_{3}\right)$ & 48.5 & 52.1320 & 0.1060 \\
\hline
\end{tabular}

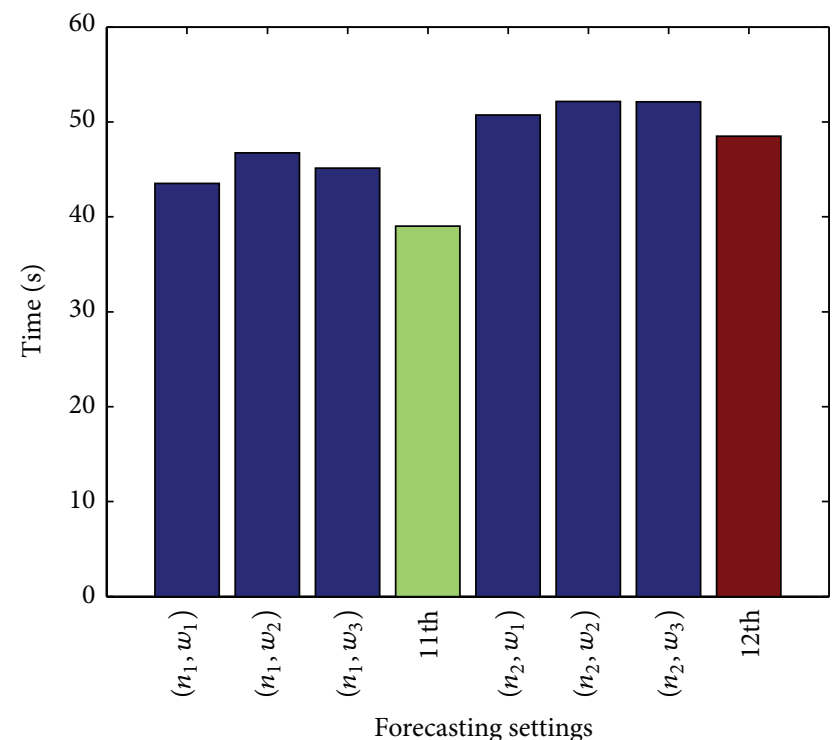

FIGURE 1: Real time performance of weighted moving average method with different parameters.

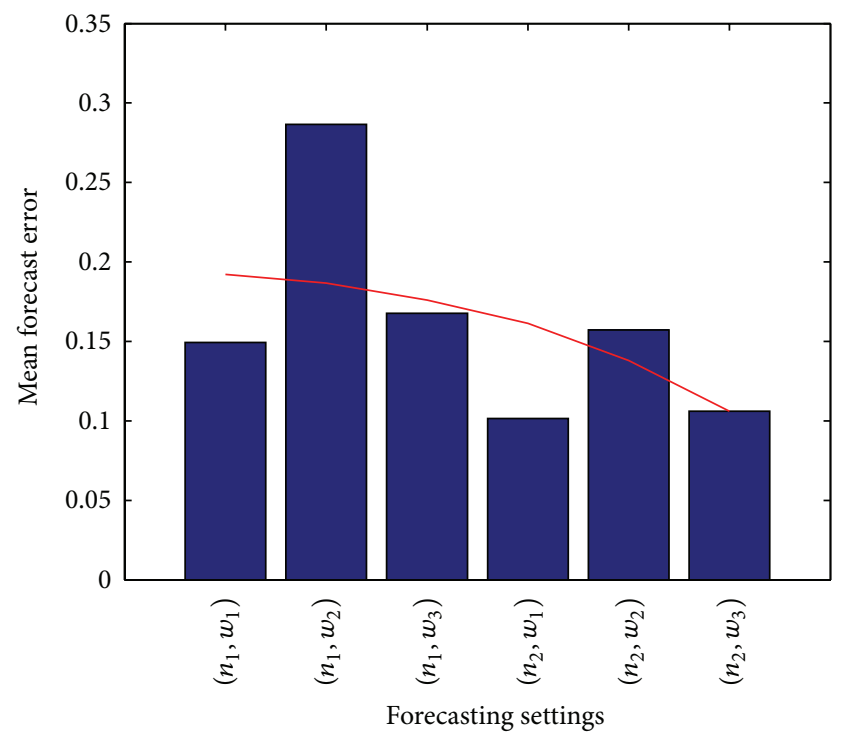

FIgURE 2: Reliability of weighted moving average.

Exponential smoothing forecasting method requires only two values: the latest one on measured and predicted values. Prediction of events is as follows:

$$
\mathrm{ES}_{t+1}=S_{w} x_{t}+\left(1-S_{w}\right) \mathrm{ES}_{t}
$$




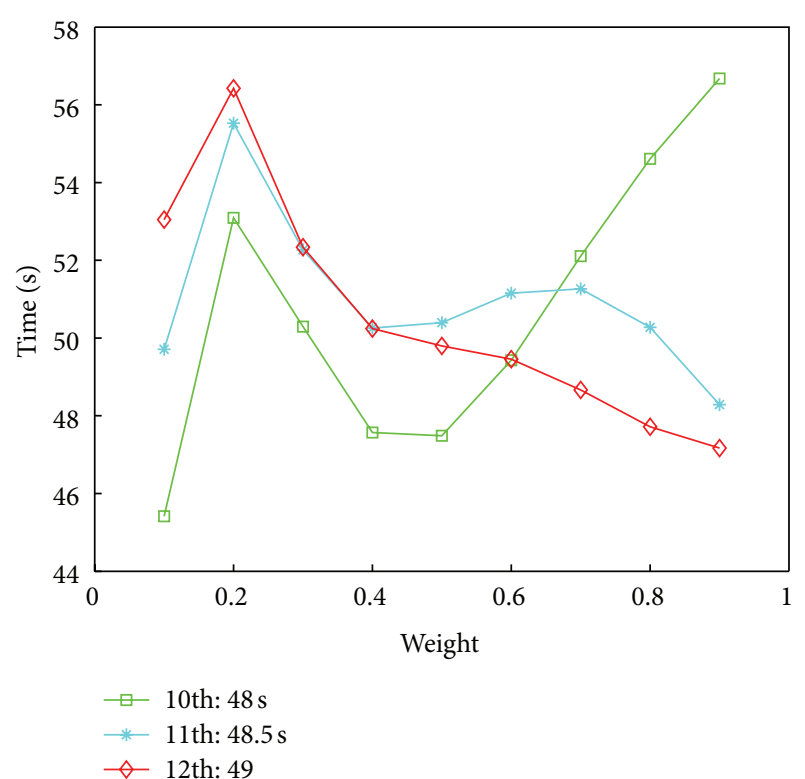

FIGURE 3: Analyses of reliability and real time with exponential smoothing prediction.

Here, $\mathrm{ES}_{t+1}$ denotes the predicting value after $t$ cyclical movements and $S_{w}$ denotes smooth weights.

Then, the $n+1$ and $n+2$ packet loss time would be predicted according to $n$ statistical values, which is used to evaluate the reliability and real time performance of this approach. $S_{w}$ is set from 0.1 to 1 . The results are shown as in Figure 3.

From Figure 3, the following was found.

(1) $S_{w}$ should be less than 0.5 when the 10 th was predicted based on 9 actual values, and there is an optimal value.

(2) When the 11th and 12th time were predicted, $S_{w}$ should be greater than 0.5 , and there is an optimal value.

(3) In the later events, the best $S_{w}$ should be closer to 1.

In summary, weighted moving average method could choose the best $n$ and $w$ based on the prediction request, but there are the past statistics and enough storage space. Exponential smoothing prediction could predict the event trend considering and sensing the reliability and timeliness without past statistics. However, prediction accuracy of weighted moving average is better than the other. Since multilevel sensing combination prediction method based on supporting diversity forecast request was proposed, its workflow is given in Figure 4.

\section{Adaptive Opportunistic Cooperative Control Mechanism}

In IOT, sending sensor transports data to receiving sensor. Assuming that there are M-1 neighbor sensors, which may join the cooperative transmission, the SNR of receiving sensor could be calculated by

$$
\begin{aligned}
\operatorname{SNR}=\frac{E}{\sigma^{2}} \sum_{i=1}^{M} & \mathrm{AP}_{i} \mathrm{TP}^{i}\left|h_{i}\right|^{2} \\
& \times\left(10^{\mathrm{TP}^{0}-\mathrm{TP}\left(d_{0}\right)+10 \beta \log _{10} d_{0}-\mathrm{TP}_{n}-\left(2 R_{\text {radio }} \ln _{2} P_{b} / B_{N}\right)}\right)^{-1} .
\end{aligned}
$$

Here, $E$ denotes the energy consumption of sending data at sending sensor, $\sigma^{2}$ denotes Gaussian white noise variance, AP is the activity probability of sensors, which is set from 0 to 1 , TP is the transmitting power of sensors, $h_{i}$ represents the channel quality between sensor $i$ and receiving sensor, $d_{0}$ denotes near-earth reference distance of sensors, $R_{\text {radio }}$ denotes transmitted signal speed of sending sensor, $P_{b}$ is bit error rate, and $B_{N}$ denotes noise bandwidth. The outage probability $P_{\text {out }}$ could be calculated by

$$
P_{\text {out }}= \begin{cases}0, & \mathrm{SNR} \geq \mathrm{TH}_{\mathrm{SNR}}, \\ 1-\frac{\mathrm{SNR}}{\mathrm{TH}_{\mathrm{SNR}}}, & \mathrm{SNR}<\mathrm{TH}_{\mathrm{SNR}} .\end{cases}
$$

Here, $\mathrm{TH}_{\mathrm{SNR}}$ represents the threshold of SNR.

According to formulas (5) and (6), Figure 5 gives the analysis results of SNR and $P_{\text {out }}$ with $M, \mathrm{AP}, d$, and TP, the threshold values of which could be obtained and are given as follows.

(1) Threshold of AP and $M$ : (AP $=1, M=2),(\mathrm{AP}=0.8$, $M=3),(\mathrm{AP}=0.6, M=4)$, and $(\mathrm{AP}=0.4, M=5)$.

(2) Threshold of $d$ and $M:(M=5, d \geq 10),(M=4$, $d \leq 8),(M=3, d \leq 6)$, and $(M=2, d \leq 4)$.

(3) Threshold of TP and $M:(M=5, \mathrm{TP}=3),(M=4$, $\mathrm{TP}=5),(M=3, \mathrm{TP}=7)$, and $(M=2, \mathrm{TP}=10)$.

Therefore, the adaptive opportunistic cooperative transmission control mechanism (CF-OCT) was proposed, which is based on the multilevel sensing combination prediction approach and thresholds. The system architecture is illustrated in Figure 6.

Afterwards, the basic idea of the CF-OCT is presented and its implementation at sending sensor and relay sensors is illustrated as follows in detail.

Sending Sensor. (1) The values of $E, d_{0}$, and $R_{\text {radio }}$ could be obtained with the physical and electrical properties of sensors. $P_{b}, B_{N}$, and $\sigma^{2}$ could be obtained by actual measurement or statistics.

(2) Network state is defined as $\langle$ APs, Ds, TPs, Ms $\rangle$. Here, APs denote the array of AP. Similarly, Ds, TPs, and Ms denote array of them, respectively. Then the channel state signal sequences are sent.

(3) Initialization of level I sensing decision: let AP be the control event sequence X. Steps from (4) to (7) will be executed.

(4) Randomness_Test $(\mathrm{X})$ is called, which is used to test whether the even sequence $\mathrm{X}$ has the randomness and the best 


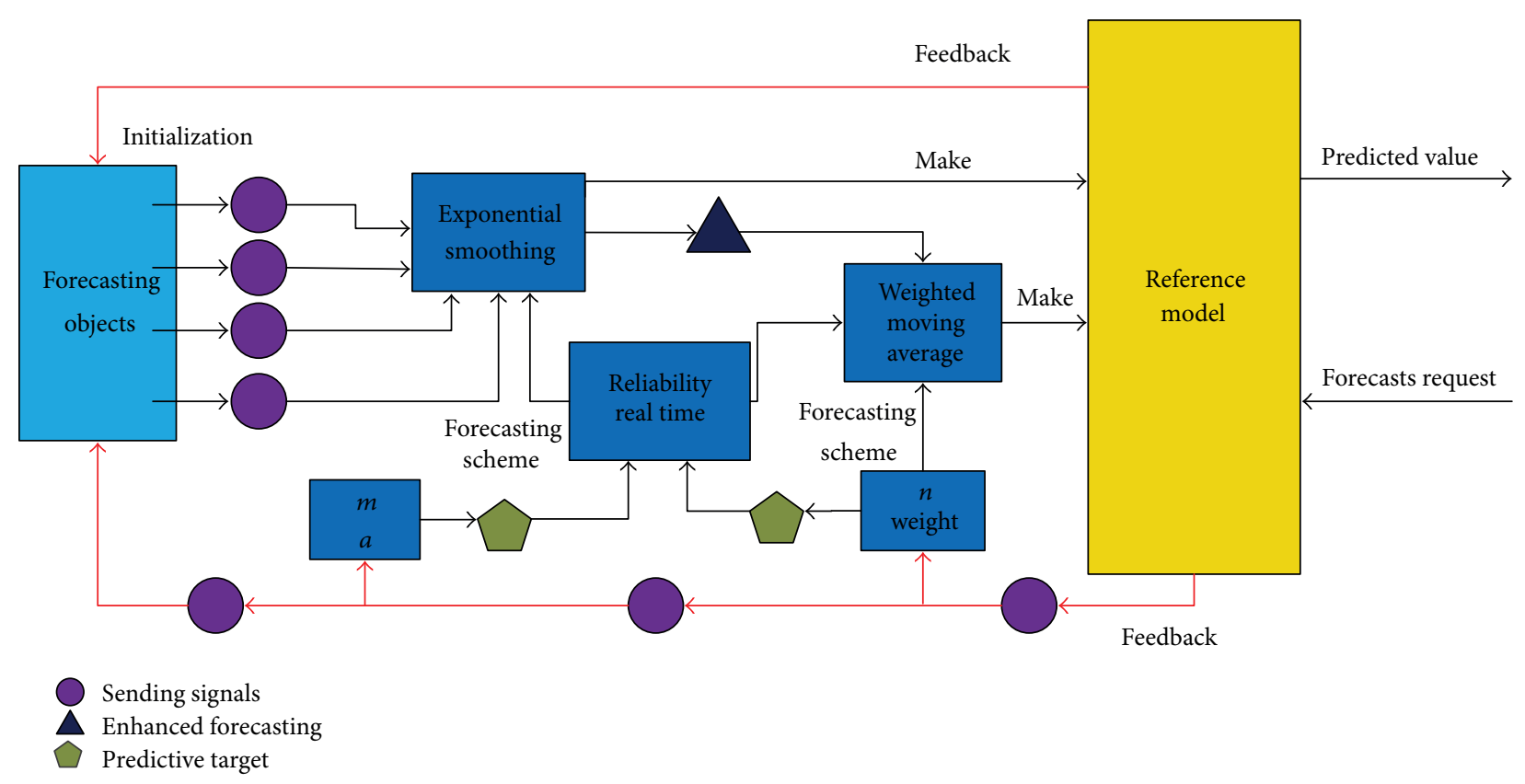

FIGURE 4: Workflow of the multilevel sensing combination forecasting method.

value of $m$ and $\partial$. Here, Randomness_Test $(\mathrm{X})$ is the function of dynamic characteristics of the events sequence detection method.

(5) Detecting whether the statistics are stored in the past: if there are these data, the step (6) will be executed. Otherwise, detecting whether there are enough storage space, the step (7) will be executed when there is not enough space. If it satisfies the condition, the step (6) will be executed.

(6) The WeightedMA(X) will be called, which is used to predict the event trend by the weighted moving average method with the best $n$ and $w$ based on sensing the prediction requirements. Here, WeightedMA $(\mathrm{X})$ is the function of weighted moving average method.

(7) The ExponentialS $(\mathrm{X})$ will be called, which is used to predict the event trend by the exponential smoothing with the best obtained $S_{w}$ by multilevel sensing approach. Here, ExponentialS(X) is the function of exponential smoothing method.

(8) The THA(AP) will be called, which is used to calculate the activity probability threshold $\mathrm{AP}_{j}(1<j>N)$. Similarly, the THA $(M)$ will be called for obtaining the cooperative sensor number threshold $M_{j}(1<j>N)$. Here, THA $(Y)$ is the function of calculating the threshold value of $Y$, which could be AP, $M, D$, or TP.

(9) Carry out level I perceive decisions. The opportunistic cooperative transmission control scheme will be created based on selecting the $M$ sensors, AP of which is larger than AP threshold.

(10) If data sending sensor receives the feedback of system or cooperative sensors, level II decision is perceived. The step (11) will be executed.

(11) Initialization of level II sending decision: let $D$ be the control event sequence X. The steps from (4) to (7) will be executed.
(12) The THA $(D)$ will be called which is used to calculate the distance threshold $D_{j}(1<j>N)$. Obtain the cooperative sensor number threshold $M_{j}(1<j>N)$ by calling THA $(M)$.

(13) Carry out level II sensing decisions. The opportunistic cooperative transmission control scheme will be created based on selecting the $M$ sensors, $D$ of which is larger than $D$ threshold.

(14) If data sending sensor receives the feedback of system or cooperative sensors, level III sensing decision is perceived. The step (15) will be executed.

(15) Initialization of level III sending decision: let TP be the control event sequence X. The steps from (4) to (7) will be executed.

(16) The THA(TP) will be called which is used to calculate the transmitting power threshold $\mathrm{TP}_{j}(1<j>N)$. Obtain the cooperative sensor number threshold $M_{j}(1<j>N)$ by calling THA $(M)$.

(17) Go in level III sensing decisions. The opportunistic cooperative transmission control scheme will be created based on selecting the $M$ sensors, TP of which is larger than TP threshold.

Cooperative Sensors. (18) Network state is defined at cooperative sensors as $\langle\mathrm{APs}, D \mathrm{~s}$, TPs, $M \mathrm{~s}\rangle$. Then the channel state signal sequences are sent.

(19) If this sensor has been selected by level I sensing decision, the step (20) will be executed. If it has been selected by level II sensing decision, the step (21) will be executed. Otherwise, the step (22) will be executed.

(20) Send the feedback to sending sensor when its AP is smaller than AP threshold.

(21) Send the feedback to sending sensor when its $D$ is smaller than $D$ threshold. 


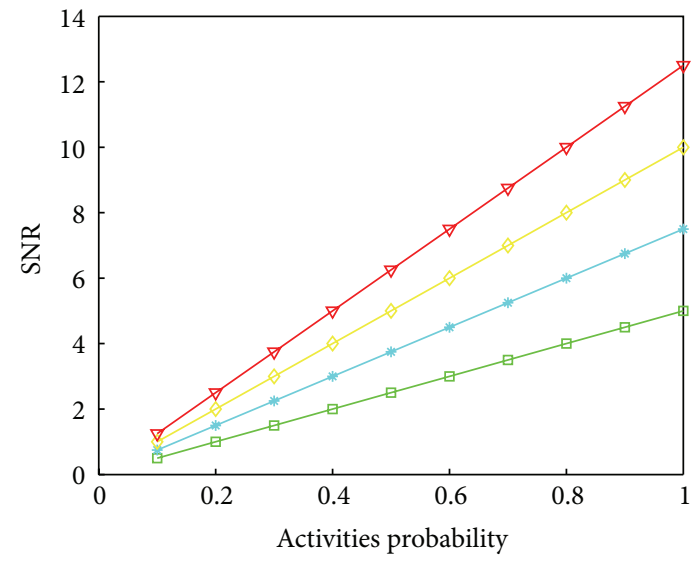

(a) SNR with AP

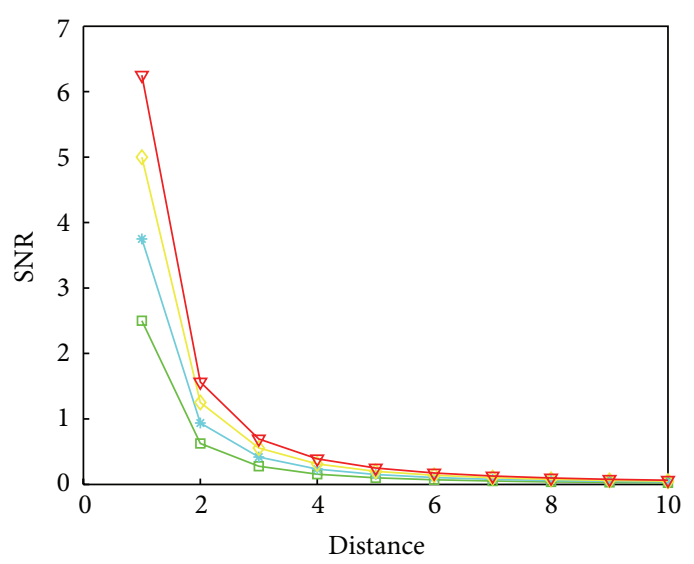

(c) SNR with $d$

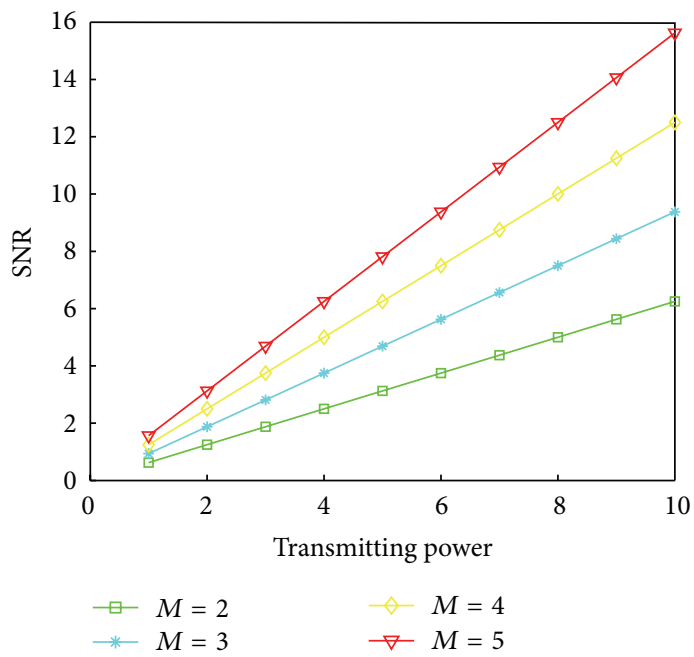

(e) SNR with TP

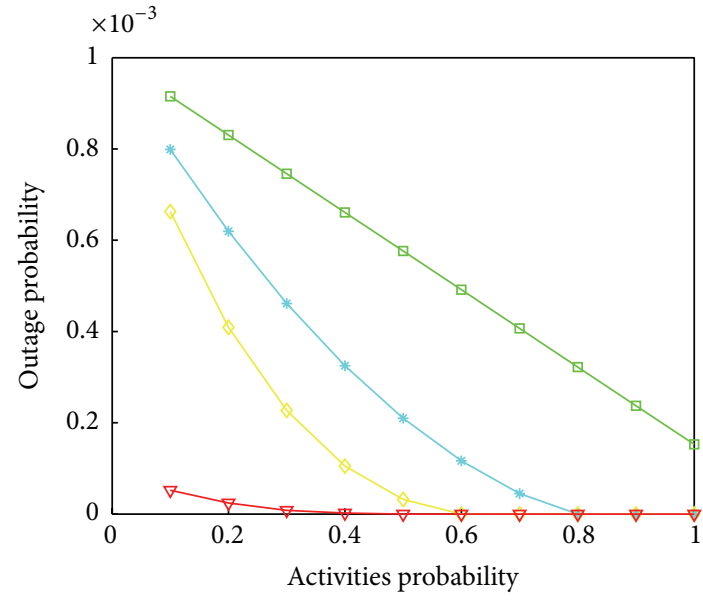

(b) $P_{\text {out }}$ with AP

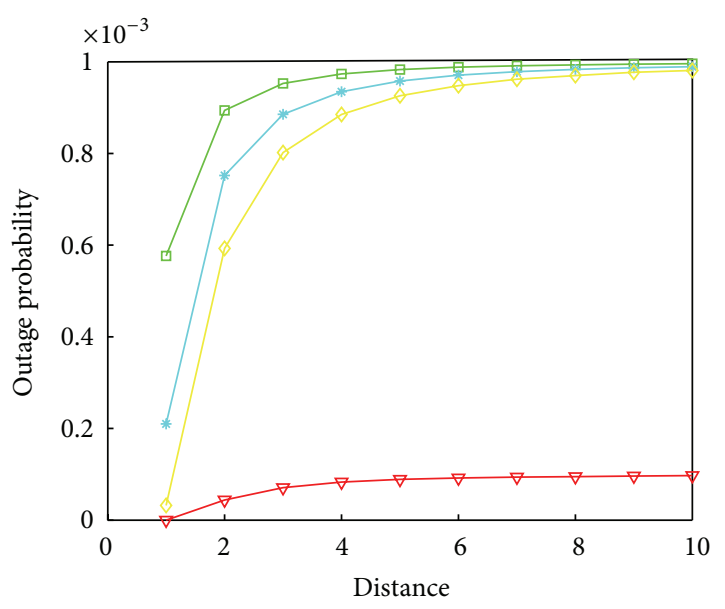

(d) $P_{\text {out }}$ with $d$

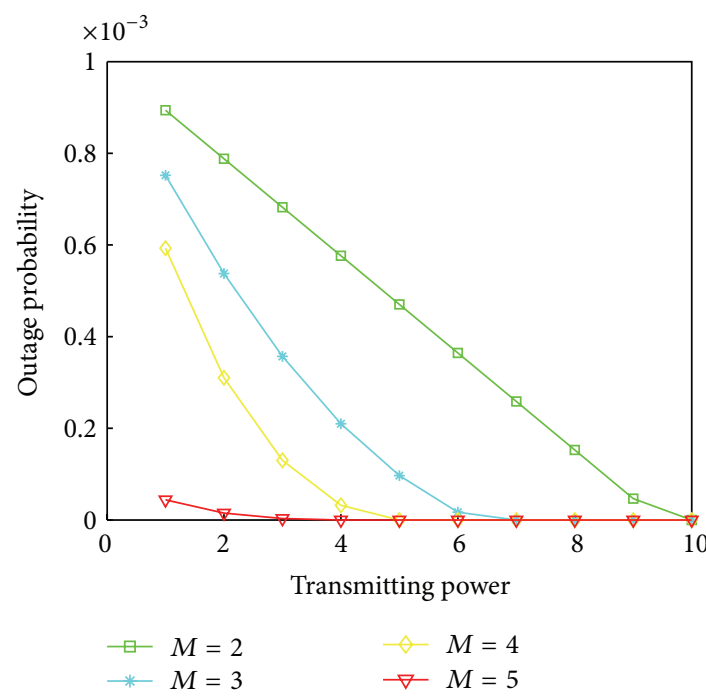

(f) $P_{\text {out }}$ with TP

FIgURe 5: Performance analysis with SNR and $P_{\text {out }}$. 


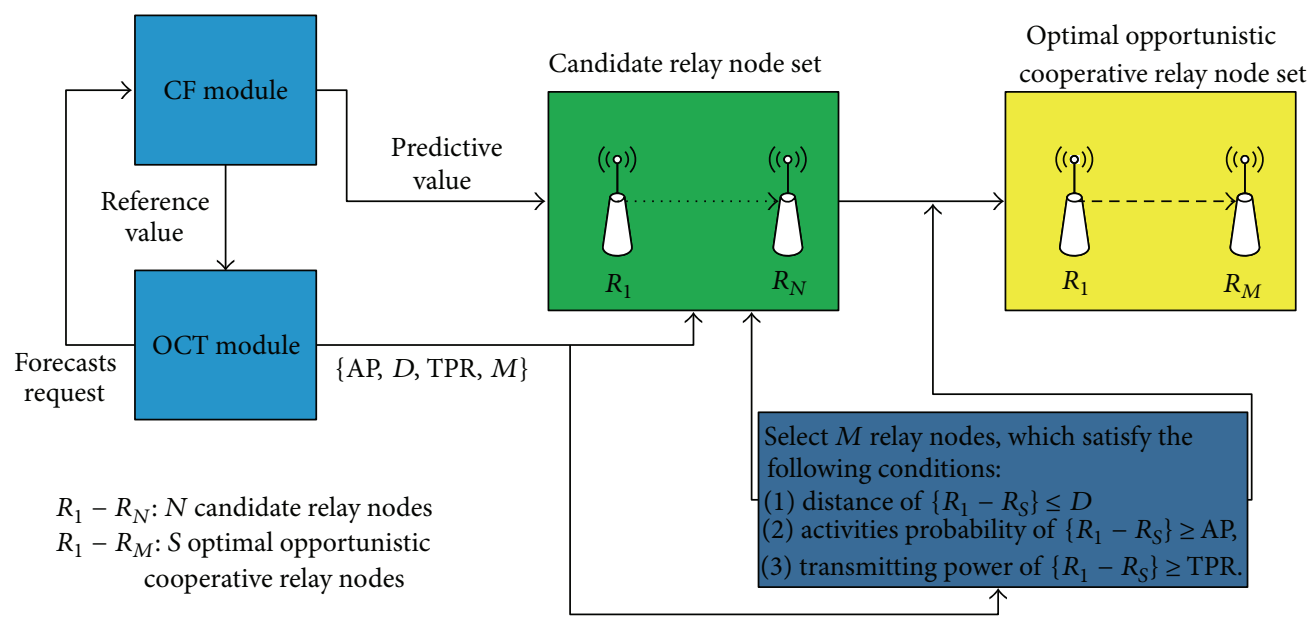

Figure 6: CF-OCT in IOT.

(22) Send the feedback to sending sensor when its TP is smaller than TP threshold.

\section{Performance Evaluation}

In this work, we simulate, analyze, and evaluate the performance of direct transmission (DT), opportunistic cooperative transmission (OCT), and the proposed CF-OCT based on activity probability. The parameter settings and environment condition are shown as in Table 3 .

The main metrics for performance evaluation for supporting the application services of mobile Internet of Things are listed as follows.

(1) Delay: the end to end delay between sending sensor and receiving sensor will be calculated merely.

(2) Throughput: statistics of data packets size received successfully at receiving sensor.

(3) Energy efficiency: ratio of energy of received data successfully to total energy consumption.

Figure 7 shows four performance metrics as a function of activity probability. The result indicates that activity probability and sensing approach have significant impact on quality of data transmission in mobile Internet of Things. We can observe tremendous improvement of performance using CFOCT, as compared with DT and OCT. Even at a lower activity of sensors or channel quality of the mobile Internet of Things channel, the performance of CF-OCT always keeps good condition, while the delay and packet error rate with DT and OCT reduce and are almost close to zero. The throughput and energy efficiency of them continue to increase and be close to 1 gradually. The reasons are that not only could the combination forecast method predict the event control trend based on multilevel sensing to minimize the end to end delay, but also the adaptive opportunistic cooperative control strategy improves the energy efficiency, which could create the optimal transmission control scheme; as a result, the system throughput utilization increased and the reliability improved.
TABLE 3: Parameter settings.

\begin{tabular}{lc}
\hline Parameters & Value \\
\hline$B_{N}$ & $30 \mathrm{kHz}$ \\
$T$ & $1 \mathrm{~s}$ \\
Moving speed & From $0 \mathrm{~m} / \mathrm{s}$ to $10 \mathrm{~m} / \mathrm{s}$ \\
$R_{\text {radio }}$ & $38.4 \mathrm{kBaud}$ \\
Mobility model & Random waypoint model \\
$d_{0}$ & From $15 \mathrm{~m}$ to $30 \mathrm{~m}$ \\
\hline
\end{tabular}

\section{Conclusions}

There is great potential for data transmission control event prediction and opportunistic cooperative control to improve the performance of mobile Internet of Things diversity services. The target of this research work is to overcome all kinds of limitations of sensors in mobile Internet of Things; we propose the adaptive opportunistic cooperative transmission control mechanism based on multilevel sensing combination prediction approach.

The main contributions in this work are as follows. First, considering the characteristics of different forecasting methods, we introduce the combination prediction approach based on multilevel sensing of sensors in mobile Internet of Things. Second, the opportunistic cooperative control strategy with diversity threshold values is designed to ensure that data packets have the optimal transmission path and control progress. Finally, we present the adaptive opportunistic cooperative mechanism, working together with above combination prediction approach, in order to improve the communication performance and guarantee the quality of services in mobile Internet of Things.

The mathematics results demonstrate that, compared with the existing typical direct transmission and opportunistic cooperation, the proposed mechanism greatly improves the data transmission quality and achieves significant gains in terms of real time performance, reliability, throughput, and energy efficiency. As a result, the proposed mechanism 


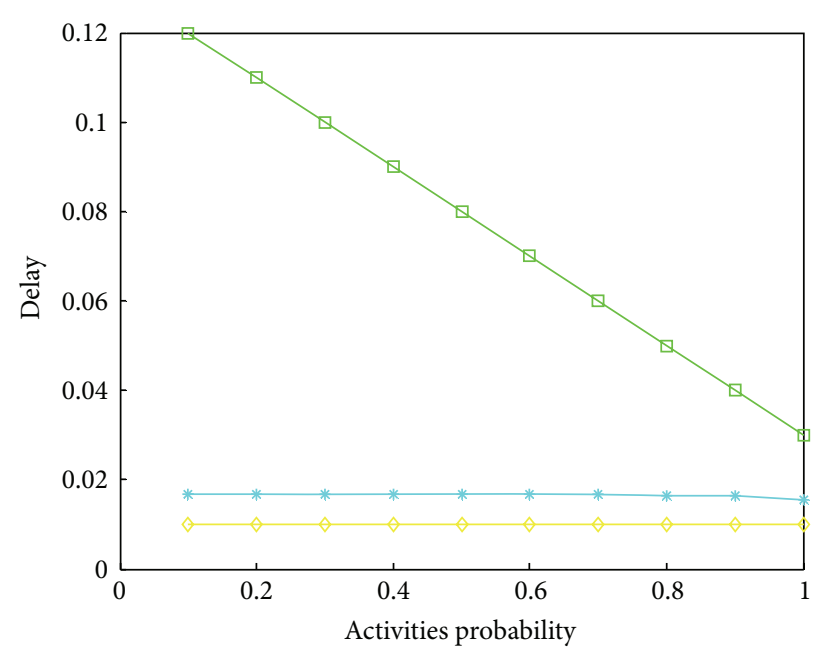

(a) Delay

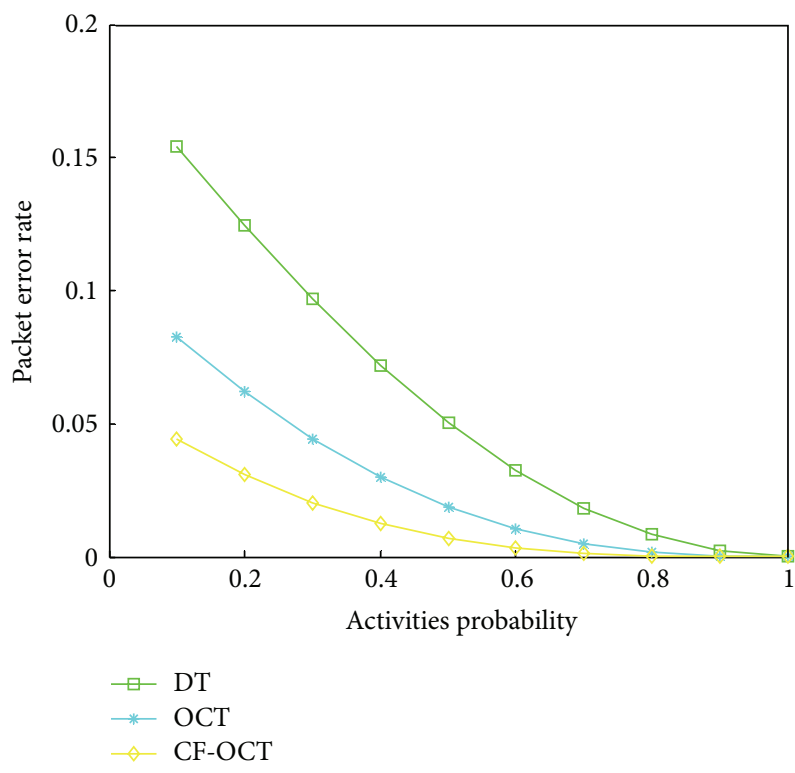

(c) Packet error rate

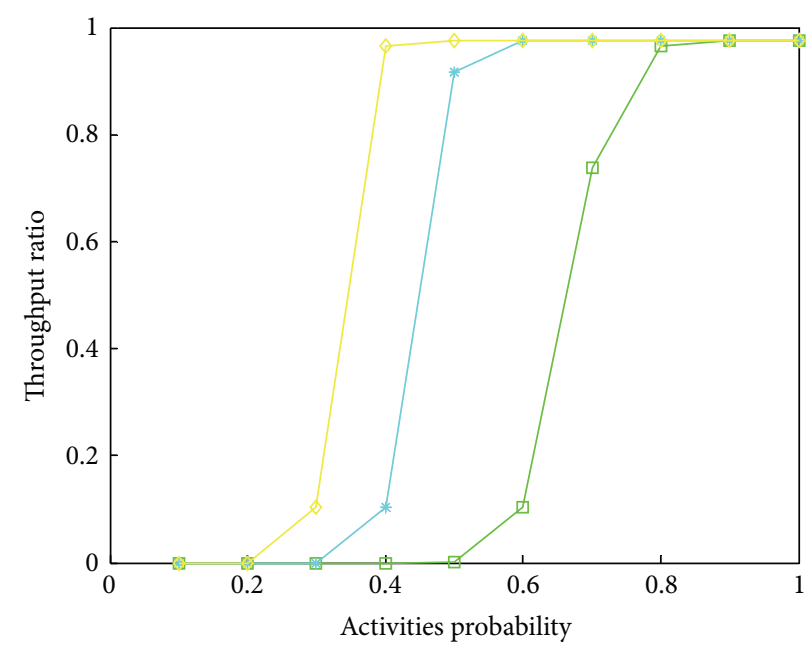

(b) Throughput

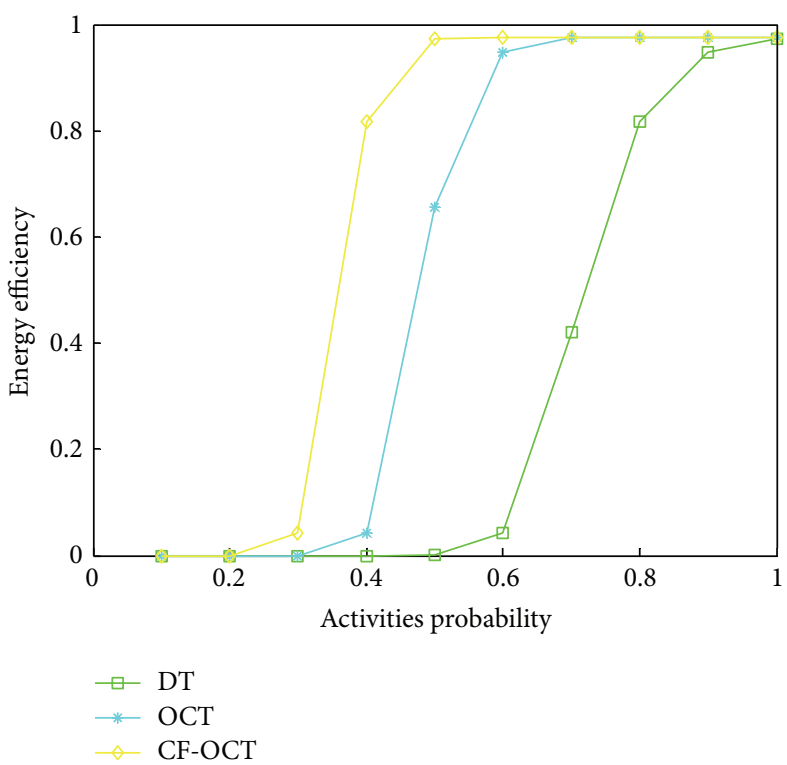

(d) Energy efficiency

Figure 7: Performance with DT, OCT, and CF-OCT.

was determined to be effective and feasible for data diversity communication in mobile Internet of Things.

\section{Conflict of Interests}

The authors declare that they have no financial and personal relationships with other people or organizations that can inappropriately influence their work; there is no professional or other personal interests of any nature or kind in any product, service, and/or company that could be construed as influencing the position presented in, or the review of, the paper.

\section{Acknowledgment}

This work is supported in part by technology projects of Changshu Municipal Health Bureau under Grant no. csws201303.

\section{References}

[1] S. Hong, D. Kim, M. Ha et al., "SNAIL: an IP-based wireless sensor network approach to the Internet of things," IEEE Wireless Communications, vol. 17, no. 6, pp. 34-42, 2010.

[2] L. Hu, Z. Zhang, F. Wang, and K. Zhao, "Optimization of the deployment of temperature nodes based on linear programing in the internet of things," Tsinghua Science and Technology, vol. 18, no. 3, pp. 250-258, 2013.

[3] Y. Kawamoto, H. Nishiyama, Z. M. Fadlullah, and N. Kato, "Effective data collection via Satellite-Routed Sensor System (SRSS) to realize global-scaled internet of things," IEEE Sensors Journal, vol. 13, no. 10, pp. 3645-3654, 2013.

[4] C. Perera, A. Zaslavsky, P. Christen, and D. Georgakopoulos, "Context aware computing for the internet of things: a survey," IEEE Communications Surveys and Tutorials, vol. 16, no. 1, pp. 414-454, 2014. 
[5] P. Bellavista, G. Cardone, A. Corradi, and L. Foschini, "Convergence of MANET and WSN in IoT urban scenarios," IEEE Sensors Journal, vol. 13, no. 10, pp. 3558-3567, 2013.

[6] Y. Wu, Q. Z. Sheng, H. Shen, and S. Zeadally, "SNAIL: an IP-based wireless sensor network approach to the internet of things," IEEE Transactions on Parallel and Distributed Systems, vol. 24, no. 10, pp. 2036-2045, 2013.

[7] V. M. Rohokale, N. R. Prasad, and R. Prasad, "A cooperative internet of Things (IoT) for rural healthcare monitoring and control," in Proceeding of the 2nd International Conference on Wireless Communication, Vehicular Technology, Information Theory and Aerospace and Electronic Systems Technology, Wireless (VITAE '11), pp. 1-6, Chennai, India, March 2011.

[8] H. Chen, D. Xiang, W. Qin et al., "A study of regional cooperative emergency care system for ST-elevation myocardial infarction patients based on the internet of things," in Proceedings of the IEEE 14th International Conference on e-Health Networking, Applications and Services (Healthcom '12), pp. 73-77, October 2012.

[9] B. Christophe, "Managing massive data of the internet of things through cooperative semantic nodes," in Proceedings of the 6th IEEE International Conference on Semantic Computing (ICSC '12), pp. 93-100, Palermo, Italy, September 2012.

[10] J. Kim and J. Lee, "Cluster-Based Mobility Supporting WMN for IoT Networks," in Proceedings of the IEEE International Conference on Green Computing and Communications (GreenCom '12), pp. 700-703, November 2012.

[11] N. Maalel, E. Natalizio, A. Bouabdallah, P. Roux, and M. Kellil, "Reliability for emergency applications in internet of things," in Proceedings of the IEEE International Conference on Distributed Computing in Sensor Systems (DCoSS '13), pp. 361-366, Cambridge, Mass, USA, May 2013.

[12] J. Niu, R. Zhang, G. Wang et al., "Design and experimental evaluation of long-distance and high-mobility ZigBee transceivers for WSNs," in Proceedings of the IEEE/CIC International Conference on Communications in China-Workshops (CIC/ICCC '13), pp. 142-147, 2013.

[13] W. A. Jabbar, M. Ismail, R. Nordin et al., "Evaluation of energy consumption in multipath OLSR routing in Smart City applications," in Proceedings of the IEEE Malaysia International Conference on Communications (MICC '13), pp. 401-406, 2013.

[14] Y. Jin, "High reliable relay selection approach for QoS provisioning in wireless distributed sensor networks," International Journal of Distributed Sensor Networks, vol. 2013, Article ID 216478, 11 pages, 2013.

[15] Y. Jin and G. Bai, "Energy-aware adaptive cooperative FEC protocol in MIMO channel for wireless sensor networks," Journal of Electrical and Computer Engineering, vol. 2013, Article ID 891429, 9 pages, 2013.

[16] B. Guo, D. Zhang, Z. Wang, Z. Yu, and X. Zhou, "Opportunistic IoT: exploring the harmonious interaction between human and the internet of things," Journal of Network and Computer Applications, vol. 36, no. 6, pp. 1531-1539, 2013.

[17] D. Rosárioa, Z. Zhao, A. Santosc et al., "A beaconless opportunistic routing based on a cross-layer approach for efficient video dissemination in mobile multimedia IoT applications," Computer Communications, vol. 45, no. 1, pp. 21-31, 2013. 

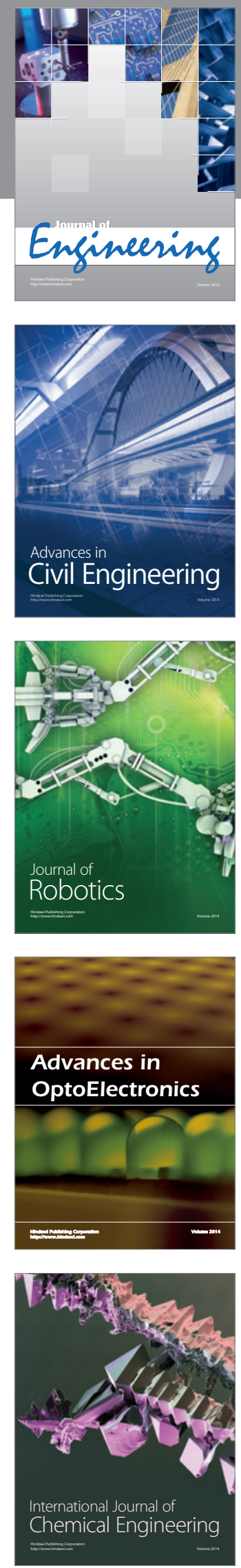

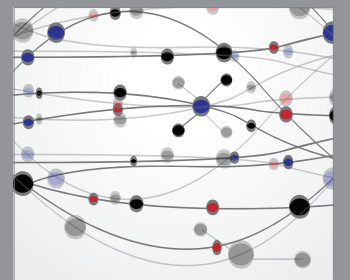

The Scientific World Journal
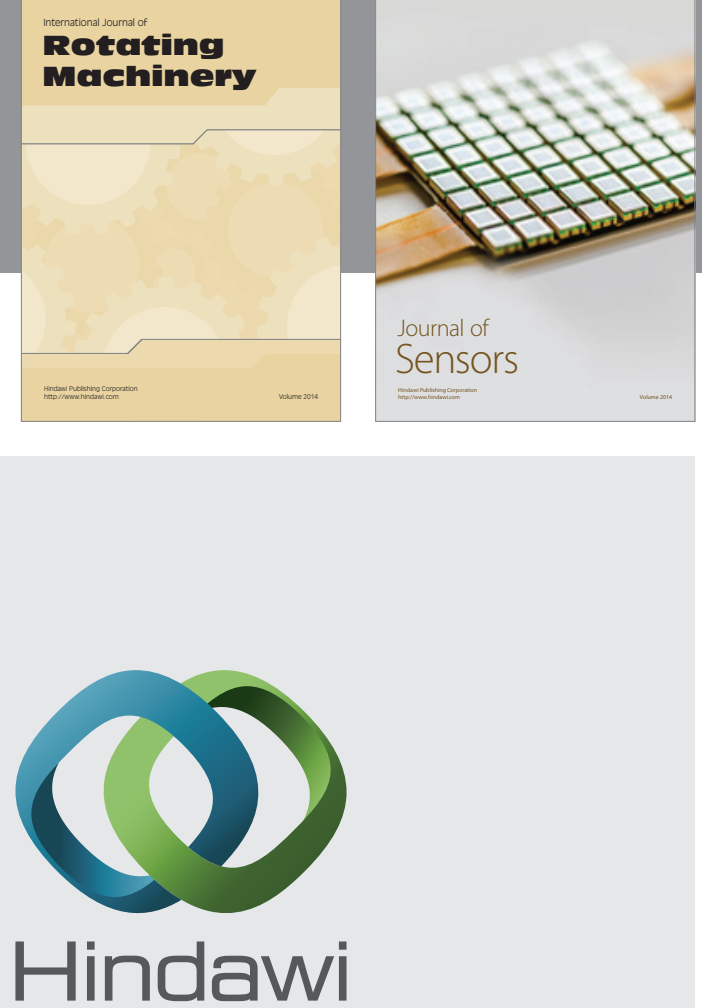

Submit your manuscripts at http://www.hindawi.com
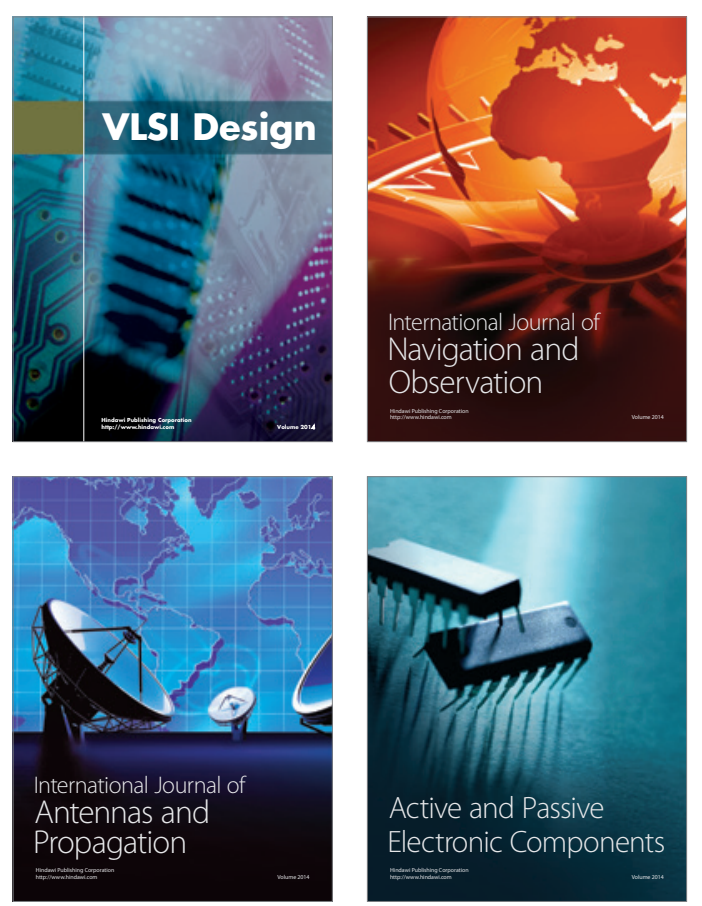
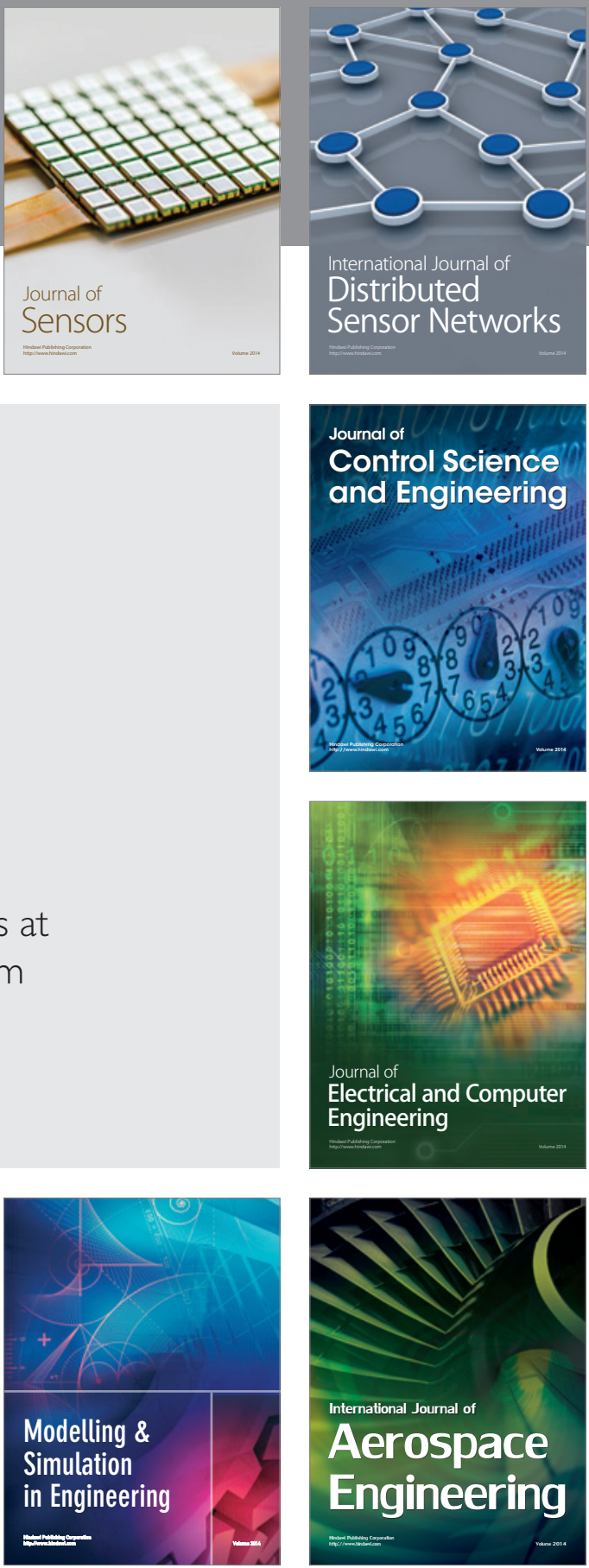

Journal of

Control Science

and Engineering
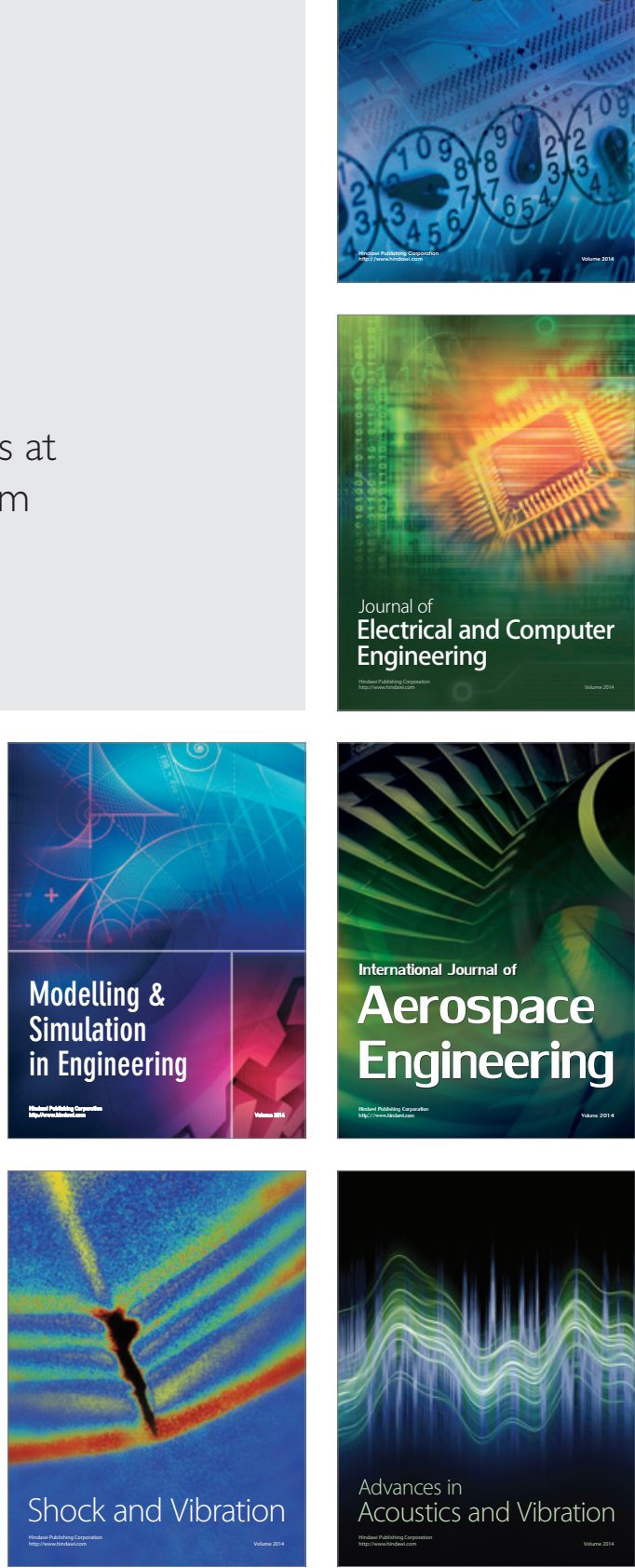